\begin{tabular}{|c|c|c|c|c|}
\hline \multirow[b]{2}{*}{$\begin{array}{l}\text { Site } \\
\text { of } \\
\text { injury }\end{array}$} & \multirow[t]{2}{*}{$\begin{array}{l}\text { Number } \\
\text { of } \\
\text { patients }\end{array}$} & Flexor injury & \multicolumn{2}{|c|}{ Intact flexor } \\
\hline & & $\begin{array}{l}\text { (confirmed at } \\
\text { operation) }\end{array}$ & $\begin{array}{l}\text { (confirmed at } \\
\text { operation for } \\
\text { associated } \\
\text { nerve injury) }\end{array}$ & $\begin{array}{l}\text { (confirmed at } \\
1 \text {-week follow-up) }\end{array}$ \\
\hline Zone 1 & 6 & 2 & - & 4 \\
\hline Zone II & 9 & 7 & 2 & - \\
\hline Zone III & 7 & 5 & - & 2 \\
\hline Zone IV & 1 & 1 & - & - \\
\hline Zone V & 10 & 4 & 4 & 2 \\
\hline
\end{tabular}

A. BANERJEE

Accident and Emergency Department, East Birmingham Hospital,

\section{Renal colic and examination of the urine in the accident and emergency department}

Examination of the urine is a routine investigation in patients presenting to the accident and emergency (A\&E) department with a suspected urinary calculus. However, there appears to be little agreement on whether urgent microscopy should be performed routinely or if dipstick urinalysis is sufficient.

Haematuria is associated with the presence of urinary calculi ${ }^{1}$ and may be extremely useful in helping to make the diagnosis before intra-venous urogram (IVU) or ultrasound examination is carried out.

In a retrospective study of 33 patients presenting to the Westminster hospital A\&E department who were diagnosed as having a urinary calculus by the A\&E senior house officer $26(79 \%)$ patients had a dipstick urinalysis and 19 (58\%) had urine microscopy. Dipsticks used were Boehringer Mannheim BM-Test $5 \mathrm{~L}$ and microscopy was with a X32 magnification inverted microscope.

Of 14 cases having both urinalysis and microscopy there were three cases of positive dipsticks and negative microscopy and one case of a negative dipstick and positive microscopy.
Nine patients were shown to have evidence of calculus on the IVU of which eight out of eight dipstick tests were positive for blood and four out of five urine microscopies were positive. Eight cases had evidence of calculus on plain abdominal radiograph and did not have an emergency IVU, of those, seven out of eight dipsticks were positive and four out of five microscopies were positive.

There were 10 patients who either had no evidence of calculus on abdominal radiograph or had a negative IVU or ultrasound. Surprisingly in this group only two out of six dipsticks were negative and two of six microscopies were negative. Only one of these patients was later proved to have had a urinary tract infection on culture, having a positive dipstick and negative microscopy.

The above small retrospective study indicates that microscopy is no more specific or sensitive than dipstick urinalysis in producing positive results for haematuria in patients who are shown to have evidence of calculus on IVU, ultrasound or abdominal radiograph. Using chemstrip 9 and $\mathrm{N}$-Multistix, Moore \& Robinson ${ }^{2}$ found that these dipsticks had an overall sensitivity of $100 \%$ and specificity of $99.3 \%$ in the detection of microhaematuria. Kennedy et al. ${ }^{3}$ found the urinary dipstick to be a safe, accurate and reliable screening test for the presence of haematuria in patients sustaining abdominal trauma. The evidence seems to indicate that urgent urine microscopy is not necessary in cases of renal colic although all patients should have urine sent for culture. Urinalysis is a simple, cheap, quick and easy test to perform in an A\&E department. Microscopy is more expensive although difficult to price accurately. A prospective study into the manage- 
ment of renal colic in the A\&E department, including the examination of the urine, is presently being undertaken.

N. EYNON-LEWIS \& D. POTTS Department of Surgery, Derriford Hospital, Plymouth PL6 8DH, UK

\section{REFERENCES}

1. Siegel Y., Korczak D., Asherov J. \& Lindner A. (1989) Renal colic and renal calculus. Harefuah 116(3), 143-145.

2. Moore G.P. \& Robinson M. (1988) Do urine dipsticks reliably predict microhaematuria? The bloody truth Annals of Emergency Medicine 17(3), 257-260.

3. Kennedy T.J., McConnell J.D. \& Thal E.R. (1988) Urine dipstick vs. microscopic urinalysis in the evaluation of abdominal trauma. Journal of Trauma 28(5), 615-617.

\section{Screening for social needs in accident and emergency departments}

It is estimated that $10 \%$ of patients attending accident and emergency (A\&E) departments are elderly and that in some cases the department is used as an extension of a primary care facility. ${ }^{1}$ In a recent report we showed that even 'minor fractures' significantly impaired elderly patients' ability to live independently and that more than $50 \%$ of such patients discharged from A\&E had functional scores compatible with moderate to severe functional impairement. ${ }^{2}$ While many of the patients acquired help from family, friends and neighbours, a proportion required additional help or were totally dependent on statutory help. This 'at risk' group needs to be identified before discharge from A\&E.

In a survey to establish the extent of social screening in A\&E; 105 patients aged 70 years or over, who had sustained minor fractures, had not been admitted to hospital, but were attending a fracture clinic were interviewed by the author during their first fracture clinic visit. The interviews occurred mostly within 7 days of sustaining a fracture $-84 \%$ were interviewed within 5 days of their injury. The patients were asked whether enquiries had been made concerning their social needs, who made such enquiries, and if they knew how to request statutory social services in the community.

We found that in this cohort of 105 patients 71 $(68 \%)$ had not been asked whether they could cope with activities of daily living once discharged. Of the remaining $34(32 \%)$ who remembered being asked an A\&E doctor; nine by a general practitioner; and three had been asked by other persons, e.g. physiotherapist.

Two patients had been interviewed by a medical social worker before leaving the A\&E department. Of patients, $42(40 \%)$ did not know how they would go about requesting statutory help in the community and $61(58 \%)$ were knowledgeable about community services.

These results mirror the findings of Currie et al..$^{3}$ who found scanty documentation of social and functional screening after scrutiny of $A \& E$ records. The problem is further amplified by the fact that $40 \%$ of the elderly patients in this study were not aware of how to acquire statutory help in the community. While the A\&E doctor may be well-placed to assess the social and functional needs of the patients, in a busy A\&E department, time is at a premium. Additionally, functional assessment requires special knowledge and skills which are not taught routinely to doctors. The importance of social management in elderly people cannot be over-emphasized and it is imperative that all who treat them be made aware of this.

To alleviate this problem, some A\&E departments have adjacent short-stay wards and cover from a social worker. Others have an 'aftercare officer' to follow-up selected patients. ${ }^{4}$ Many manpower-hours would be required to follow-up all the elderly patients considered at risk once discharged from A\&E departments to their home. The awareness of social services facilities may be enhanced by a targeted education programme, and the pick-up rate of patients at risk increased by the use of a screening questionnaire. ${ }^{5}$ It appears that the main problem lies in early and efficient identification of the patient at risk in A\&E departments. An alternative may be the placement of a key worker with special functional assessment skills as well as local social management networks to review all elderly patients who are considered at risk prior to discharge from the A\&E department. Referral to such key workers would be open, i.e. nurses, doctors and relatives would all be encouraged to enlist their services. This key worker would not only be invaluable in complementing the A\&E staff, but would also partly absorb the role of the aftercare officer whose home visiting role is already incorporated in the duties of the primary care team. This would not only prevent unscheduled return visits by patients ${ }^{6,7}$ but would also enhance the quality of life in the community.

This discussion assumes sufficient maripower, financial provision and an effective community- 